\title{
Mapping of QoS between UMTS and WiMAX in Tight Coupling Heterogeneous Wireless Network
}

\author{
Charles Sarraf ${ }^{1},{ }^{*}$ Firas Ousta ${ }^{2}$, Nidal Kamel ${ }^{3}$, Mohd Zuki ${ }^{4}$ \\ ${ }^{1}$ Touch, Beirut-Lebanon \& Holly Sprit University of Kaslik (usek), Kaslik, Lebanon \\ ${ }^{2}$ Department of Electrical and Electronic Engineering, Universiti Teknologi PETRONAS, \\ Bandar Seri Iskandar, Perak, Malaysia \\ ${ }^{3,4}$ Department of Electrical and Electronic Engineering, Universiti Teknologi PETRONAS, \\ Bandar Seri Iskandar, Perak, Malaysi \\ 1'cmsarraf@teleco-plus.com,fousta@teleco-plus.com, ${ }^{3}$ nidalkamel@petronas.com.my, \\ ${ }^{4}$ mzuki_yusoff@petronas.com.my
}

Abstract. 4G network or Heterogeneous wireless access network will integrate existing wireless access technologies (e.g. 2G, 3G, WiMAX, WLAN, etc...) in order to provide, transparently, endusers with the "best" service through connecting their mobile devices at any time to the best available radio network. In that context, mapping the QoS classes between these different access networks becomes a big challenge. In this paper, we propose a method of mapping between the CoS of UMTS and QoS categories of WiMAX in a tight coupling architecture of Heterogeneous Wireless Access Network (HWAN).

Keywords: Quality of Services (QoS), 4G Network, Heterogeneous Wireless Access Network (HWAN), Next Generation Wireless Network (NGWN)

\section{Introduction}

In the last few decades, various wireless and mobile networks (e.g. 2G, 3G, WiFi, WiMax, cdma, cdma2000, etc...) have been developed and deployed across the world. These networks have different access technologies and have been designed to work independently without cooperating with each other. Seamless interworking between these wireless networks, either directly or over a common IPbased backbone, as illustrated in Figure 1, is an ultimate objective of the upcoming Heterogeneous Wireless Access Network (HWAN), also known as Next Generation Wireless Network (NGWN) and referred to as $4 \mathrm{G}$ (Fourth Generation) wireless network; that, in addition to the integration of the already existing wireless networks, should provide new access technologies (e.g. LTE-Advanced and WirelessMAN-Advanced) that will offer higher bandwidth (100Mbps - 1Gbps).

One of the major challenge to (HWAN) and 4G wireless network is to support QoS [1] due to the different channel characteristics, various access controls, varying bit rate, bandwidth allocation methods, fault tolerant levels and handoff methods, protocols and supports. QoS support can occur at access level, packet level, transaction level, circuit level, core network and connectivity level as well as user level. In a complete wireless solution, the End-to-End communication between two or multiple users will likely involve multiple wireless networks, with different types of accesses, as well as the underlying IP-based networks.

This paper addresses the issues of supporting QoS in tight coupling approach of $4 \mathrm{G}$ wireless networks from End-to-End point of view and presents novel mapping mechanisms between UMTS CoS and WiMAX QoS categories in tight coupling approach, however it does not address the other 


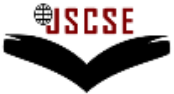

DOI: 10.7321/jscse.v2.n3.1

issues and challenges neither mapping the QoS between the other wireless access networks nor in loose coupling.

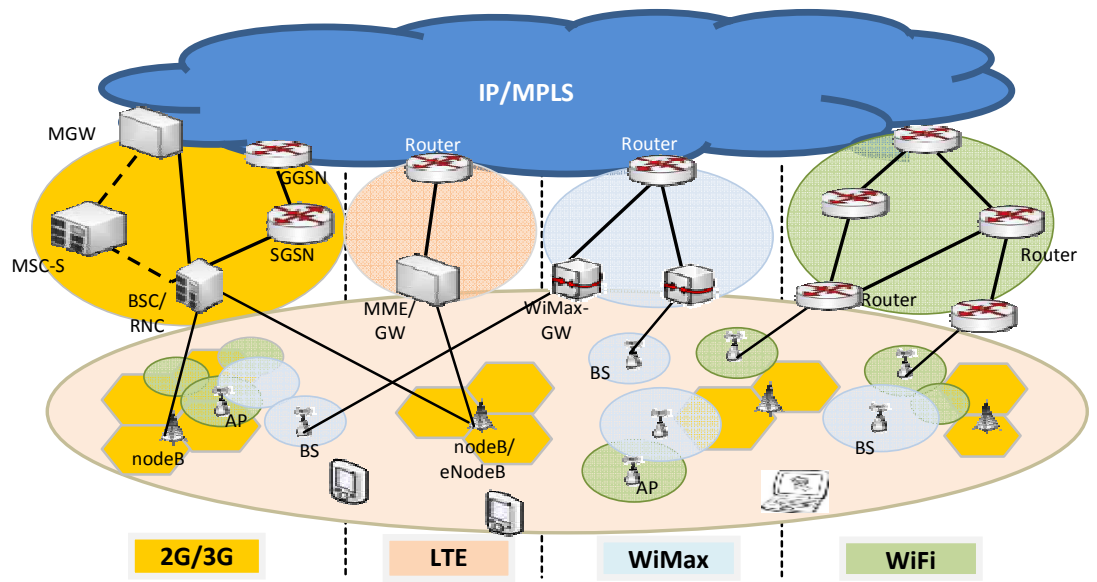

Figure 1. Heterogeneous Wireless Network Architecture

The structure of this paper is as follows, section 2 tangles the open issues and challenges of Heterogeneous Wireless Access Network in general and in tight coupling in particular; section 3 describes the architecture of Heterogeneous wireless network with its two methods: loose coupling and tight coupling; Overview of QoS is described in section 4; while a novel methods for mapping CoS of UMTS into WiMAX QoS categories with their associated parameters is presented in section 5; and finally section 6 concludes by summing up with emphasis on related future work.

\section{Challenges and issues}

This integration will definitely bring many challenges; such as Security (e.g. authentication and authorization), Accounting and Billing, Access Control, Handoff, service differentiation and End-toEnd QoS [1]. Also connecting these different wireless technologies, either directly or over a common IP-based backbone, and getting them to work together is also a major challenge. In order to offer the mobile users transparent and seamless services; ranging from traditional voice to VoIP and multimedia applications through web browsing, chatting, on-line gaming, the "best" available radio technology should be used at anytime and anywhere.

As mentioned earlier, these wireless access technologies have different characteristics that have been designed for different purposes but can complement each other. Cellular systems (e.g. 2G, cdma and $3 \mathrm{G}$ ) provide wide coverage areas, full mobility and roaming, traditionally focusing on voice and offering low bandwidth and limited data support. In the last few years, these networks have been developed (GPRS, EDGE, UMTS, HSPA, HSPA+, LTE, 1xEV-DO, etc...) to offer better data support with higher rates reaching 100Mbps. However, this comes at high cost because they still support two types of access, circuit switched for voice and video calls and packet switched for data and IP traffic.

On the other hand, WLANs and WiMax, with only packet switched access method, provide high data rate at low cost. While WLANs, which are organized in form of hotspots, provide limited coverage in specific areas, WiMAX can supply mobile broadband for anyone, anywhere [2]. They are both expected to provide access to IP-based services, including VoIP and multimedia services, at high data rates.

As mentioned earlier, one of the major issues and challenges, to $4 \mathrm{G}$ wireless networks, is supporting End-to-End QoS and providing the end users with the QoS that are promised to get regardless of the access technology that being used. Methods and mechanisms that may be used to support the various 


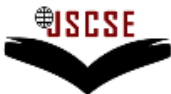

DOI: 10.7321/jscse.v2.n3.1

issues and challenges, as presented earlier in this section in general and QoS in particular, vary based on the type of coupling. While gateways may be used in the case of loose coupling, modifications to protocols, interfaces, and services and some nodes of existing radio networks are needed in tight coupling as described in section 3 .

\section{Heterogeneous Network Architecture}

The 4G wireless network will integrate existing and new radio access networks that will work together to transparently offer the mobile users the need services with the corresponding QoS requirements. This will definitely bring several advantages for all parties including mobile users, network providers and service providers. On one hand, users will benefit from the coverage and characteristics provided by the different networks by allowing them to seamlessly connect, at any time and any place, to the access network that offers the "best" possible quality and the most economical service, while network and service providers will deploy the most economical technologies and infrastructures based on the offered services.

In that sense, $4 \mathrm{G}$ wireless network shall provide the mobile users with more bandwidth, better QoS at anytime and anywhere with reduced costs for both end-users and providers.

Since each access network has different levels, methods and protocols for QoS parameters (such as bandwidth, delay, jitter, bit error rates, mobility, coverage and costs); maintaining End-to-End QoS across heterogeneous wireless access networks will be one of the main challenges to $4 \mathrm{G}$ wireless networks.

Handoff methods shall enable mobile terminals to maintain connectivity when moving between cells, systems frequencies and IP subnets, allowing them to interoperate among different network regardless of the different signaling protocols, routing techniques and mobility management standards [3].

For the above reasons, the architecture of $4 \mathrm{G}$ wireless network should be flexible in a way the QoS methods and parameters should be seamlessly be mapped and/or translated as the mobile terminals are moving both horizontally and vertically across the different access technologies.

Two main strategies, interworking and integrating, have been proposed as solutions to connect the different radio networks in the heterogeneous wireless network.

In integrated networks, the air interfaces from different radio access technologies are coupled at either radio access network (RAN) or core network (CN) levels [4]. Whereas, interworking networks method is constructed by using edge gateways and linking between them to connect different wireless systems, and exchanging the information and signaling through those gateways [5, 6]. Different architectures, for both strategies, have been proposed depending on the level of interdependence. For example, the European Telecommunications Standards Institute (ETSI) specifies two methods for interconnecting WLANs with cellular networks: loose coupling and tight coupling [7].

\subsection{Loose Coupling}

The loose coupling technique depends on gateways to connect the integrated wireless via IP-based internet backbone. In this approach, there is no direct link between the different radio access networks. Additional equipment's, such as WLAN/WiMAX gateways may be needed to support authentication, QoS mapping, billing and other services [10,11]. Figure 2 illustrates a loose coupling integration between 3G/UMTS, WiMAX and WLAN networks. 


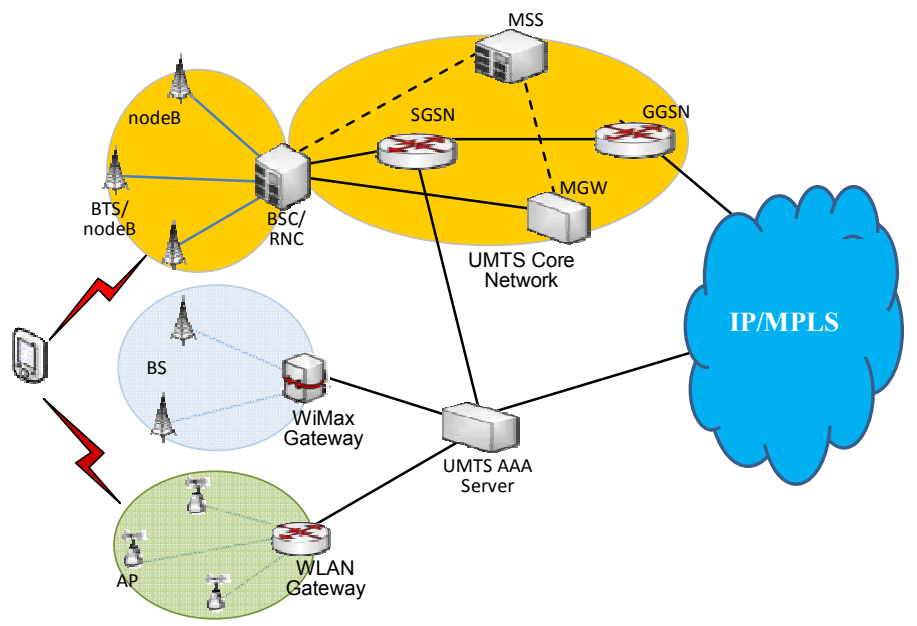

Figure 2. Loose Coupling Integration

In this example, the gateways are mainly connected to the IP-based network through a UMTS AAA (Authentication, Authorization and Accounting) server and do not have any direct connection to the UMTS network. This makes the WLAN and WiMAX additional access networks to the 3G/UMTS network. They may be owned and operated by different operators with roaming agreements, which may be used to offload the 3G/UMTS networks and provide better and cheaper services, especially in populated areas.

On the other hand, the main disadvantage is the two domains are separated. The mobility signaling may traverse a relatively long path, thus inducing relatively high handoff latency $[5,18]$ which may affect the QoS of real time applications.

\subsection{Tight Coupling}

In the tight coupling approach, on the other hand, the WLAN and WiMAX access networks are directly connected to the cellular core network, and appear, to it, as parts of the cellular radio network forming one single radio access network (RAN).

Integration between the different wireless access networks is achieved by introducing WLAN and WiMAX gateways, where the WLAN, WiMAX data traffic goes through the cellular core network before reaching the external Packet Data Networks (PDNs) [9].

In this approach, each of the existing networks must modify their protocols, interfaces, and services to meet the requirements of interworking requirements such as authentication, accounting, mobility management and QoS [8].

The main feature of the tightly coupled architecture is that handoff and security issues in WLAN and WiMAX are totally controlled by the cellular network handoff and security systems. Whereas, the main disadvantages of the tight coupling approach are as follows $[5,8]$ :

- An interface in the cellular core network exposed to WLAN/WiMAX is required, which is a challenge as the systems are likely to be developed and deployed independently by different operators.

- A large volume of WLAN/WiMAX traffic will go through the cellular core network, possibly making the latter a bottleneck.

- WLAN/WiMAX needs to have a protocol stack compatible with that of cellular networks. The induced complexity and cost may hamper the deployment of a tight coupling architecture.

Moreover, with the tight coupling approach, the integration of WLAN and WiMAX networks with $3 \mathrm{G} / \mathrm{UMTS}$ network can be made at the core network level (i.e., GGSN or SGSN) 
or at the access network level (i.e.., RNC), which are classified as tight and very tight coupling respectively $[10,11]$.

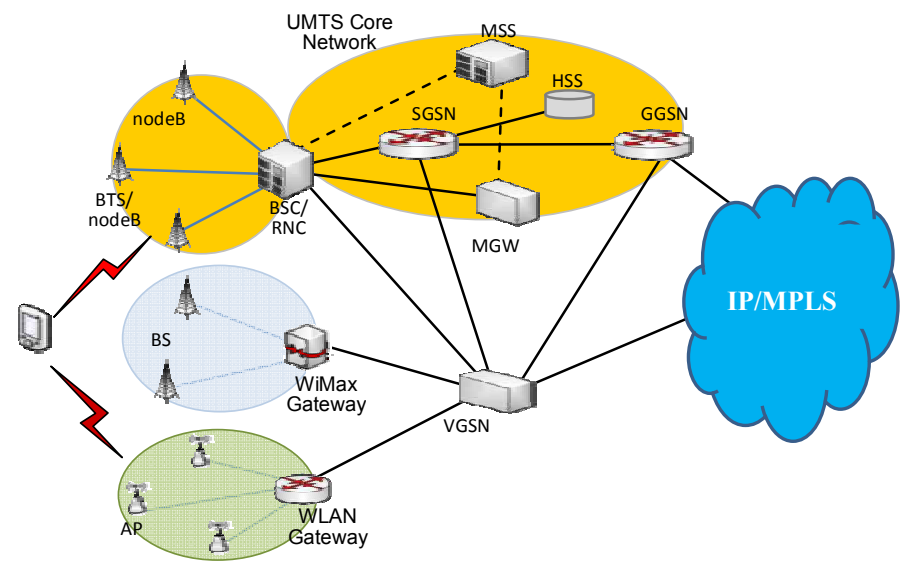

Figure 3. Tight Coupling Integration at the GGSN level

Figure 3 presents an example of tight coupling of WLAN and WiMAX with 3G/UMTS network at the GGSN level, where a logical node called virtual GPRS Support Node (VGSN) has been introduced to interconnect the WLAM and WiMAX with the 3G/UMTS network [19]. The main functionality of the newly introduced node is to exchange subscriber and mobility information and to route data traffic between the integrated networks.

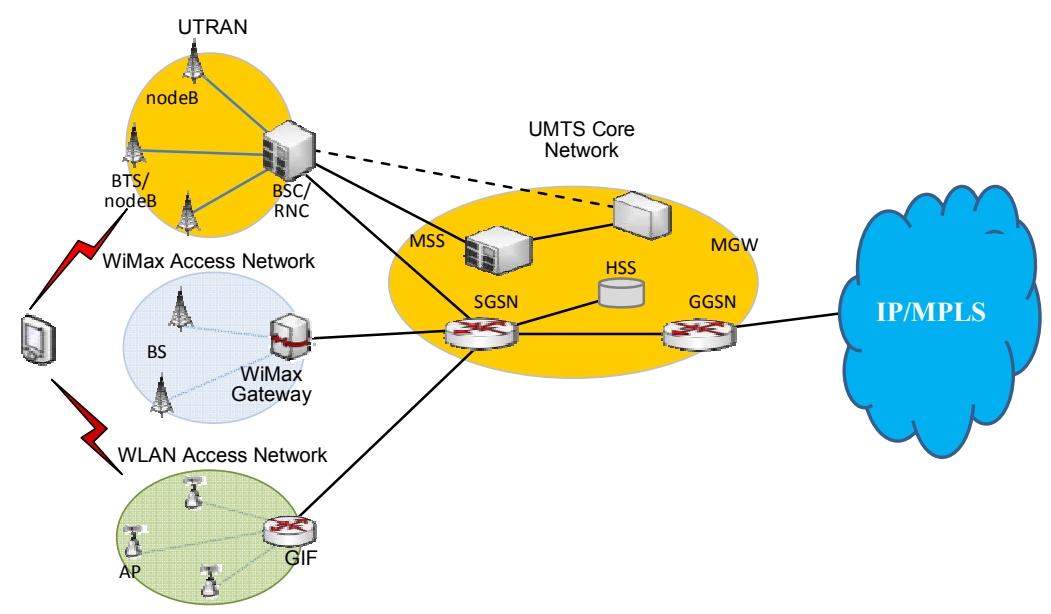

Figure 4. Tight Coupling Integration at the SGSN level

Tight coupling can be also achieved at the SGSN level as illustrated in figure 4 [9]. In this architecture, the WLAN and WiMAX networks are deployed as alternate RAN connected with the $3 \mathrm{G} / \mathrm{UMTS}$ wireless network resulting in the $3 \mathrm{G} / \mathrm{UMTS}$ core network not distinguishing between WLAN/WiMAX radio network and $3 \mathrm{G}$ radio network.

Another approach of tight coupling can be achieved at the RNC level, focusing on interworking at the UTRAN level and incorporating RNC functionality into WLAN/WiMAX components [20]. 


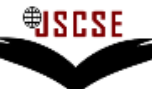

DOI: 10.7321/jscse.v2.n3.1

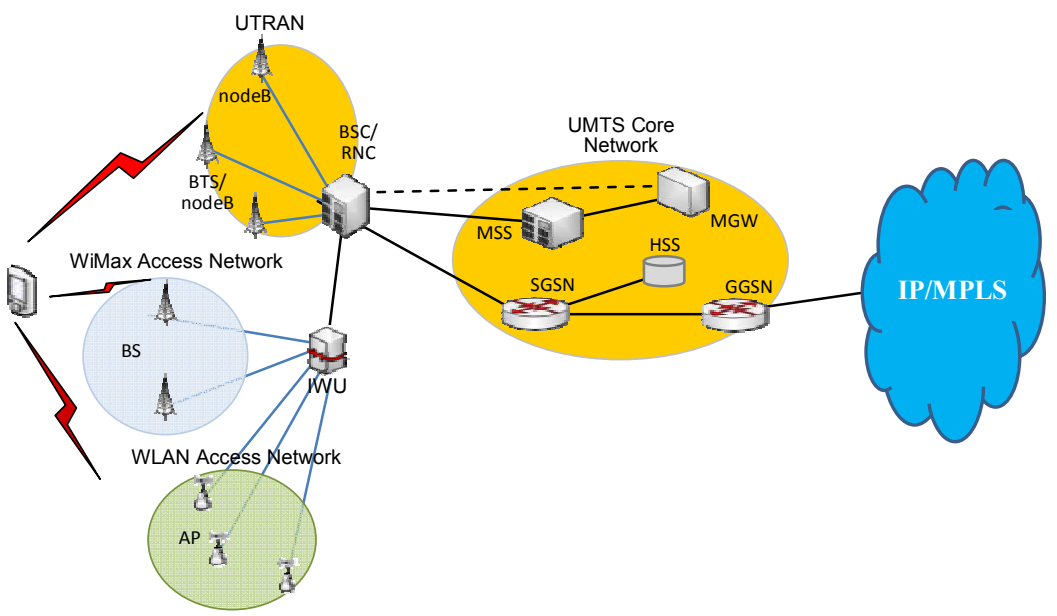

Figure 5. Tight Coupling Integration at the RNC level

Figure 5 shows an example of such architecture, where the integration is accomplished by an Interworking Unit (IWU) node which is responsible for protocol translation and signaling exchange between the RNC and WLAN (hotspots) and WiMAX (BS) access technologies.

The main advantage of this approach is its efficiency when it comes to mobility management, since it is based on existing UMTS functionalities and protocols. It also ensures the continuity of services such as authentication, authorization, accounting and billing allowing the mobile users to maintain their sessions, as they move for one network to another with the possibility of service continuation with related QoS requirements.

Generally, tight coupling integration is more complex than loose coupling. This is due to the fact that in tight coupling approach WLAN and WiMAX traffic will go through 3G/UMTS core network directly affecting the setup of the whole network. In addition to this, some design, modification and configuration of the network elements may be required to support the WLAN/WiMAX radio access integration and the increased traffic load.

\subsection{Interworking}

Both tight and loose coupling methods are defined in Third Generation Partnership Project (3GPP) as Generic Access Network (GAN) and Interworking WLAN (I-WLAN). I-WLAN is defined in [12] where the 3GPP-WLAN interworking subsystem provides bearer services allowing the $3 \mathrm{GPP}$ users to utilize the WLAN to access PS based services. However, it is referred to as loose coupling since the WLAN data do not traverse the Serving GPRS Support Node (SGSN) or the Gateway GPRS Support Node (GGSN).

Similarly, Worldwide Interoperability for Microwave Access (WiMAX) has defined many interworking strategies between WiMAX and others wireless systems. In [13], WiMAX-3GPP interworking is specified based on the I-WLAN architecture. The solution covers both Direct IP access and 3GPP IP access denoted WiMAX Direct IP access and WiMAX 3GPP IP respectively. [14] specifies seamless data mobility between WiMAX subscribers and 3GPP packet data subscribers and defines an interworking solution for Dual Radio MS that is capable of simultaneously transmitting and receiving on both WiMAX and 3G. Finally, the interworking between WiMAX and WLAN with its new logical entities are defined in [15].

On the other hand, tight coupling approach requires development and integration of WLAN/WiMAX protocol stack compatible with that of cellular networks and need to be deployed in SGSN, GGSN or 
RNC. As discussed earlier, this may affect the deployment of such an approach due to the induced complexities and costs.

\section{Quality of Services}

One of the major challenges for $4 \mathrm{G}$ wireless networks or Heterogeneous Wireless Access Networks (HWAN) is supporting End-to-End QoS which may consists of two major issues:

1. Mapping the QoS messages and their related parameters over different access/connectivity networks at registration, call setup and connections.

2. Maintaining these QoS classes when mobile users move, vertical handoff, from one access network to another access network. This may also cause changes in the backbone and connectivity's network based on the coupling approach that is in use. Whereas in tight coupling approach the backbone remains the one of the cellular network, in loose coupling transition of the already established connection is a must and could be over an external IP-based network.

Different access and connectivity networks have, through their standardization and specifications, defined different QoS protocols, messages and parameters.

\subsection{ITU}

The ITU-T has defined QoS as "the collective effect of service performance which determine the degree satisfaction of a user of the service" [21].

The ITU-T recommendation [22] focuses on four different points of view, which are represented in Figure 6:

\footnotetext{
- Customer's QoS requirements

. Service providers's offering of QoS

- QoS achieved or delivered

. Customer survey rating of QoS
}

A model for multimedia Quality of Service categories from end-user viewpoint is defined in [23].

The Recommendation also defines performance targets for audio, video and data applications by specifying typical data rates and KPIs that can be used to measure the QoS as detailed in Tables $1 \& 2$. Since these performance targets, data rates and KPIs are applications' specific, they can be applied to any type of network, radio access, and connectivity or backbone networks and therefore can be used, as reference, for End-to-End QoS, in $4 \mathrm{G}$ mobile networks.

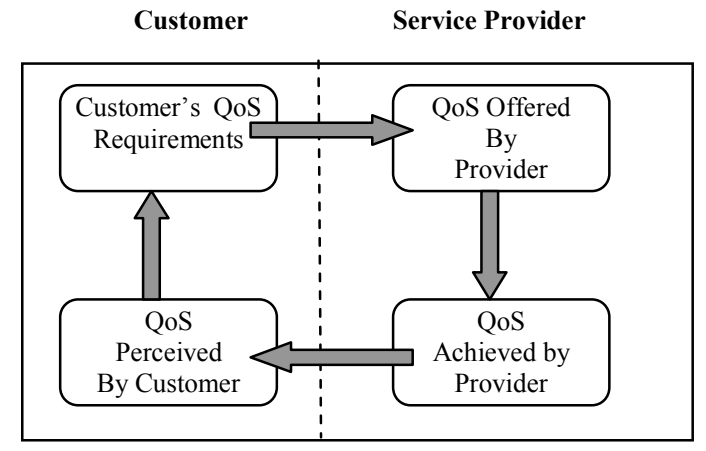

Figure 6. The four viewpoints of QoS. 
Table 1. Performance targets for audio and video applications

\begin{tabular}{|l|l|l|l|l|l|}
\hline \multicolumn{1}{|c|}{ Application } & Symmetry & Data rates & \multicolumn{3}{|l|}{ Key performance parameters \& target values } \\
\hline & & & One-way delay & Jitter & Information loss \\
\hline $\begin{array}{l}\text { Conversational } \\
\text { voice }\end{array}$ & Two-way & $4-64 \mathrm{kbit} / \mathrm{s}$ & $\begin{array}{l}<150 \mathrm{~ms} \\
<400 \mathrm{~ms} \text { limit }\end{array}$ & $<1 \mathrm{~ms}$ & $<3 \%$ PLR \\
\hline Voice messaging & $\begin{array}{l}\text { Primarily } \\
\text { one-way }\end{array}$ & $4-32 \mathrm{kbit} / \mathrm{s}$ & $\begin{array}{l}<1 \mathrm{~s} \text { for playback } \\
<2 \mathrm{~s} \text { for record }\end{array}$ & $<1 \mathrm{~ms}$ & $<3 \%$ PLR \\
\hline $\begin{array}{l}\text { High quality } \\
\text { streaming audio }\end{array}$ & $\begin{array}{l}\text { Primarily } \\
\text { one-way }\end{array}$ & $16-128 \mathrm{kbit} / \mathrm{s}$ & $<10 \mathrm{~s}$ & $<1 \mathrm{~ms}$ & $<1 \%$ PLR \\
\hline Videophone & Two-way & $16-384 \mathrm{kbit} / \mathrm{s}$ & $\begin{array}{l}<150 \mathrm{~ms} \text { preferred } \\
<400 \mathrm{~ms} \text { limit }\end{array}$ & & $<1 \%$ PLR \\
\hline Video - One-way & One-way & $16-384 \mathrm{kbit} / \mathrm{s}$ & $<10 \mathrm{~s}$ & & $<1 \%$ PLR \\
\hline
\end{tabular}

\subsection{UMTS}

3GPP provided frameworks for QoS within UMTS [24] and End-to-End QoS involving GPRS [25], specifying a list of attributes applicable to UMTS Bearer Service and Radio Bearer Service, and describing the Quality of Service architecture to be used in UMTS networks [24]. It also defines the interaction between the TE/MT Local Bearer Service, the GPRS Bearer Service, the external Bearer Service, and how these together provide Quality of Service for the End-to-End Service [25]. These frameworks also define the IP level mechanisms necessary to provide End-to-End QoS involving GPRS networks, including possible interaction between the IP level and the GPRS level, as well as the application level as depicted in Figure 9.

The End-to-End Service on the application level uses the bearer services of the underlying network(s) and could be conveyed over several networks (not only UMTS). Therefore, the End-to-End QoS used by TE will be realized using TE/MT Local Bearer Service, a UMTS Bearer Service, and an External Bearer Service.

Four QoS classes, corresponding to different traffic QoS requirements are defined in [25]. They are:

- Conversational class. Audio and video communications with real time constraints.

- Streaming class. This class comprises applications for downloading multimedia contents (audio and video) that are reproduced on-line.

- Interactive class. Remote access applications, in which a human or a machine sends requests to a distant server and waits for an answer in a "reasonable" time.

- Background class. A whole range of data applications for which delay is not a concern.

Each one has different QoS attributes used by network elements for prioritization, scheduling and queuing. However, the most important QoS parameters used to differentiate traffic are Maximum Bit Rate (MBR), Guaranteed Bit Rate (GBR), Traffic Handling Priority (THP) and Allocation/Retention Priority (ARP). 
Table 2. Performance targets for data applications

\begin{tabular}{|l|l|l|l|l|l|}
\hline \multicolumn{1}{|c|}{ Application } & Symmetry & \multicolumn{1}{|c|}{$\begin{array}{c}\text { Amount of } \\
\text { data }\end{array}$} & \multicolumn{2}{|c|}{ Key performance parameters and target } \\
values
\end{tabular}

In more detail, each PDP context has a QoS profile formed by UMTS bearer and Radio Access Bearer attributes (RAB). These attributes are classified as Traffic Class, Maximum bit rate (MBR), Guaranteed bit rate (GBR), Delivery order, Maximum service data unit (SDU), SDU service information, SDU error ratio, Residual bit error ratio, Delivery of erroneous SDUs, Transfer delay, Traffic handling priority (THP), Allocation/Retention priority (ARP) and Source statistic descriptor.

Moreover, within the same QoS class, further differentiation may be provided to diverse services using a different bit rate or ARP [16]. The most important QoS parameters which are used to differentiate traffic in UMTS classes of service are listed in Table 3.

Table 3. UMTS Class of Services

\begin{tabular}{|l|l|l|l|l|}
\hline Traffic Class & ARP & THP & MBR & GBR \\
\hline Conversational & Yes & No & Yes & Yes \\
\hline Streaming & Yes & No & Yes & Yes \\
\hline Interactive & Yes & Yes & Yes & No \\
\hline Background & Yes & No & Yes & No \\
\hline
\end{tabular}

A list of UMTS attributes are also defined as well as mapping these attributes to the different classes. This is presented in Table 4 [24]. The value ranges reflect the capability of the UMTS network. 
Table 4. Value ranges of the UMTS bearer service attributes

\begin{tabular}{|l|l|l|l|l|}
\hline Traffic class & $\begin{array}{l}\text { Conversational } \\
\text { class }\end{array}$ & Streaming class & Interactive class & $\begin{array}{l}\text { Background } \\
\text { class }\end{array}$ \\
\hline $\begin{array}{l}\text { Maximum bitrate } \\
\text { (kbps) }\end{array}$ & $<=16000$ & $<=16000$ & $\begin{array}{l}<=16000- \\
\text { overhead }\end{array}$ & $\begin{array}{l}<=16000- \\
\text { overhead }\end{array}$ \\
\hline Delivery order & Yes/No & Yes/No & Yes/No & Yes/No \\
\hline $\begin{array}{l}\text { Maximum SDU size } \\
\text { (octets) }\end{array}$ & $<=1500$ or 1502 & $<=1500$ or 1502 & $<=1500$ or 1502 & $<=1500$ or 1502 \\
\hline $\begin{array}{l}\text { SDU format } \\
\text { information }\end{array}$ & RAN WG3 & RAN WG3 & & \\
\hline $\begin{array}{l}\text { Delivery of erroneous } \\
\text { SDUs }\end{array}$ & Yes/No & Yes/No & Yes/No & Yes/No \\
\hline $\begin{array}{l}\text { Residual BER } \\
\text { SDU error ratio }\end{array}$ & $\begin{array}{l}5^{*} 10^{-2}, 10^{-2}, 5^{*} 10^{-3}, \\
10^{-3}, 10^{-4}, 10^{-5}, 10^{-6}\end{array}$ & $\begin{array}{l}5^{*} 10^{-2}, 10^{-2}, 5^{*} 10^{-3}, \\
10^{-3}, 10^{-4}, 10^{-5}, 10^{-6}\end{array}$ & $4^{*} 10^{-3}, 10^{-5}, 6^{*} 10^{-8}, 10^{-3}$, & $10^{-1}, 10^{-2}, 7^{*} 10^{-3}$, \\
$10^{-3}, 10^{-5}, 10^{-5}$, \\
$6^{-5}, 10^{-8}$
\end{tabular}

\subsection{WiMAX}

QoS in WiMAX networks is usually managed at the medium access control (MAC) layer. The bandwidth allocation is done based on two ways: (i) Grant Per Connection (GPC), in which bandwidth is assigned to each connection, and (ii) Grant Per Subscriber Station (GPSS), in which SS re-distributes the transmission slots allocated by the BS to all connections.

Table 5. WiMax QoS Categories

\begin{tabular}{|c|c|c|}
\hline QoS Category & Applications & QoS Specifications \\
\hline $\begin{array}{c}\text { UGS } \\
\text { Unsolicited Grant Service }\end{array}$ & VoIP, T1/E1, ATM CBR & $\begin{array}{l}\text { Maximum Sustained Rate } \\
\text { Maximum Latency Tolerance } \\
\text { Jitter Tolerance }\end{array}$ \\
\hline $\begin{array}{c}\text { rtPS } \\
\text { Real-Time Polling Service }\end{array}$ & Streaming Audio or Video & $\begin{array}{l}\text { Minimum Reserved Rate } \\
\text { Maximum Sustained Rate } \\
\text { Maximum Latency } \\
\text { Tolerance Traffic Priority }\end{array}$ \\
\hline $\begin{array}{c}\text { nrtPS } \\
\text { Non-Real-Time Polling Service }\end{array}$ & File Transfer Protocol (FTP) & $\begin{array}{l}\text { Minimum Reserved Rate } \\
\text { Maximum Sustained Rate } \\
\text { Traffic Priority }\end{array}$ \\
\hline $\begin{array}{c}\text { BES } \\
\text { Best-Effort Service }\end{array}$ & $\begin{array}{l}\text { Data Transfer, Web } \\
\text { Browsing, etc. }\end{array}$ & $\begin{array}{l}\text { Maximum Sustained Rate } \\
\text { Traffic Priority }\end{array}$ \\
\hline $\begin{array}{c}\text { ErtPS } \\
\text { Extended Real Time Polling } \\
\text { Service }\end{array}$ & $\begin{array}{l}\text { Voice with Activity } \\
\text { Detection (VoIP) }\end{array}$ & $\begin{array}{l}\text { Minimum Reserved Rate } \\
\text { Maximum Sustained Rate } \\
\text { Maximum Latency } \\
\text { Tolerance Jitter } \\
\text { Tolerance Traffic Priority }\end{array}$ \\
\hline
\end{tabular}


A service flow refers to unidirectional flow of packets that is associated with a particular QoS. The IEE802.16 standard [17] has defined five types of service flows, as detailed in Table 5, each with different QoS requirements:

1. Unsolicited Grant Service (UGS): designed to support real-time service flows that generate fixed size packets on periodic basis, such as TI, El and VoIP without silence suppression.

2. Real Time Polling Service (rtPS): designed to support real-time service flows that generate variable size data packets on a periodic basis, such as MPEG video.

3. Non-Real Time Polling Service (nrtPS): introduced for non-real-time flows which require variable size data grants on a regular basis, such as high bandwidth FTP.

4. Best Effort Service (BES): designed to support best effort traffic such as email and offers no guarantee.

5. Extended Real Time Polling Service (ErtPS): designed to support voice with activity detection such as VoIP.

\section{QoS mapping between UMTS and WiMax in tight coupling}

As described earlier, in tight coupling architecture, integrating WiMAX network in 3G/UMTS network is achieved either at the SGSN, GGSN or RNC level where mapping of the protocols, messages and parameters is performed. We propose a mapping scheme, as detailed in Table 6, for QoS classes of services and their attributes, between UMTS and WiMax. This mapping should achieve the End-to-End QoS, for the different end-users' services, as defined in the ITU-T frameworks and described in 3.1. 


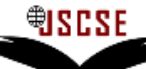

DOI: 10.7321/jscse.v2.n3.1

Table 6. Mapping between UMTS CoS and WiMAX QoS categories

\begin{tabular}{|c|c|c|c|c|}
\hline \multirow{3}{*}{ UMTS CoS } & \multicolumn{3}{|c|}{ QoS Parameters Mapping } & \multirow{2}{*}{ WiMAX QoS } \\
\hline & UMTS Attributes & $\begin{array}{c}\text { Value } \\
\text { Ranges }\end{array}$ & WiMAX Attributes & \\
\hline & Maximum hit rat (khns) & & & \multirow{5}{*}{ UGS } \\
\hline \multirow{4}{*}{$\begin{array}{l}\text { Conversational } \\
\text { Class }\end{array}$} & Maximum bit rate (kbps) & & Maxımum Sustained Rate & \\
\hline & Guaranteed bit rate (kbps) & $<=256000$ & Minimum Reserved Rate & \\
\hline & Transfer Delay (ms) & 80 max value & Maximum Latency & \\
\hline & Jitter & $<1 \mathrm{~ms}$ & Tolerance Jitter & \\
\hline \multirow{5}{*}{$\begin{array}{l}\text { Conversational } \\
\text { Class }\end{array}$} & Maximum bit rate (kbps) & $<=256000$ & Maximum Sustained Rate & \multirow{5}{*}{ ertPS } \\
\hline & Guaranteed bit rate (kbps) & $<=256000$ & Minimum Reserved Rate & \\
\hline & Transfer Delay (ms) & 80 maximum & Maximum Latency & \\
\hline & Jitter & $<=1 \mathrm{~ms}$ & Tolerance Jitter & \\
\hline & Allocation/Retention Priority & $1,2,3$ & Tolerance Traffic Priority & \\
\hline \multirow{5}{*}{ Streaming Class } & Maximum bit rate (kbps) & $<=256000$ & Maximum Sustained Rate & \multirow{5}{*}{ rtPS } \\
\hline & Guaranteed bit rate (kbps) & $<=256000$ & Minimum Reserved rate & \\
\hline & Transfer Delay (ms) & 300 Maximum & Maximum Latency & \\
\hline & Jitter & $<=1 \mathrm{~ms}$ & Tolerance Jitter & \\
\hline & Allocation/Retention Priority & $1,2,3$ & Tolerance Traffic Priority & \\
\hline \multirow{5}{*}{$\begin{array}{l}\text { Interactive } \\
\text { Class }\end{array}$} & Maximum bit rate (kbps) & $<=256000$ & Maximum Sustained Rate & \multirow{5}{*}{ nrtPS } \\
\hline & Guaranteed bit rate (kbps) & No Limit & Minimum Reserved rate & \\
\hline & Transfer Delay (ms) & No Limit & Maximum Latency & \\
\hline & Jitter & $<1 \mathrm{~ms}$ & Tolerance Jitter & \\
\hline & $\begin{array}{c}\text { Traffic Handling Priority } \\
\& \\
\text { Allocation/Retention Priority }\end{array}$ & $1,2,3$ & Tolerance Traffic Priority & \\
\hline \multirow{5}{*}{$\begin{array}{l}\text { Interactive } \\
\text { Class }\end{array}$} & Maximum Bit rate (kbps) & $<=256000$ & Maximum Sustained Rate & \multirow{5}{*}{$\mathbf{B E}$} \\
\hline & Guaranteed bit rate (kbps) & No Limit & Minimum Reserved Rate & \\
\hline & Transfer Delay (ms) & No Limit & Maximum Latency & \\
\hline & Jitter & $<1 \mathrm{~ms}$ & Tolerance Jitter & \\
\hline & $\begin{array}{c}\text { Traffic Handling Priority } \\
\& \\
\text { Allocation/Retention Priority }\end{array}$ & $1,2,3$ & Tolerance Traffic Priority & \\
\hline \multirow{5}{*}{$\begin{array}{l}\text { Background } \\
\text { Class }\end{array}$} & Maximum bit rate (kbps) & $<=256000$ & Maximum Sustained Rate & \multirow{5}{*}{ BE } \\
\hline & Guaranteed bit rate (kbps) & No Limit & Minimum Reserved Rate & \\
\hline & Transfer delay (ms) & No Limit & Maximum Latency & \\
\hline & Maximum bit rate (kbps) & No Limit & Tolerance Jitter & \\
\hline & Allocation/Retention Priority & $1,2,3$ & Tolerance Traffic Priority & \\
\hline
\end{tabular}

\section{Conclusion and future work}

In Heterogeneous Wireless Access Network, providing the applications with required QoS as Endto-End is a big challenge. A mapping scheme between the four CoS of UMTS and the five QoS categories of WiMAX, in a tight coupling architecture, has been proposed. Such mapping should untimely achieve the End-to-End QoS, for the different applications and services, as defined in the ITU-T specifications. These mapping mechanisms, with their associated messages, parameters and attributes, must be evaluated and optimized, in order to achieve the required End-to-End QoS to be offered to the mobile users in both cases: access level and vertical handoff level.

Simulation techniques can be used to test and verify the proposed mapping schemes and ultimately find the optimized values for the various parameters and attributes that will achieve End-to-End QoS. 


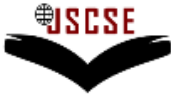

DOI: 10.7321/jscse.v2.n3.1

\section{References}

[1] C. Sarraf, F. Ousta. "End-to-End Quality of Services Issues in 4G Mobile Networks", 12th WSEAS International Conference on Communications, Crete Island, Greece, July 23-25 2008.

[2] R. Beaubrun, "Integration of Heterogeneous Wireless Access Networks", Heterogeneous Wireless Access Networks, vol. 1, 1, ed. Springer, 2008.

[3] D. G. Stratogiannis, et al., "4G Wireless Networks: Architectures, QoS Support and Dynamic Resource Management", Wireless Network Traffic and Quality of Service Support: Trends and Standards, vol. 1, T. Lagkas, et al., ed. Information Science Reference, 2010.

[4] W. Hongyi, et al., "Integrated cellular and ad hoc relaying systems: iCAR", IEEE Journal on Selected Areas in Communications, vol. 19, pp. 2105-2115, 2001.

[5] S. Wei, et al., "Resource management for QoS support in cellular/WLAN interworking", Network, IEEE, vol. 19, pp. 12-18, 2005.

[6] M.-B. Xavi, et al., "The EuQoS system: a solution for QoS routing in heterogeneous networks [Quality of Service based Routing Algorithms for Heterogeneous Networks]", Communications Magazine, IEEE, vol. 45, pp. 96-103, 2007.

[7] E. T. S. Institute, "Requirements and Architectures for Interworking between HIPERLAN/2 and 3rd Generation Cellular Systems", ETSI TR 1019572001.

[8] X. Fangmin, et al., "Interworking of Wimax and 3GPP networks based on IMS [IP Multimedia Systems (IMS) Infrastructure and Services]”, Communications Magazine, IEEE, vol. 45, pp. 144150, 2007.

[9] A. K. Salkintzis, et al., "WLAN-GPRS integration for next-generation mobile data networks", IEEE Wireless Communications, vol. 9, pp. 112-124, 2002.

[10]L. Lampropoulos, et al., "A flexible UMTS/WLAN architecture for improved network performance”, Wireless Personal Communications, vol. 43, pp. 889-906, 2007.

[11]L. Lampropoulos, et al., "Handover management architectures in integrated WLAN/cellular networks", IEEE Communications Surveys \& Tutorials, vol. 7, pp. 30-44, 2005.

[12] G. T. 23.234, "3GPP system to Wireless Local Area Network (WLAN) interworking”, ed., 3GPP, 2006.

[13] WiMax Forum, "WiMAX - 3GPP Interworking”, vol. WMF-T37-002-R010v3, ed., WiMax Forum 2008.

[14] WiMax Forum, "WiMAX Pre-Release 8 3GPP Interworking”, vol. WMF-T37-008-R016v01 ed., WiMAX Forum, 2010.

[15] WiMax Forum, "Architecture, detailed Protocols and Procedures for Wi-Fi WiMAX Interworking”, vol. WMF-T37-010-R016v01 ed., WiMAX Forum, 2010.

[16]C. Jianfeng, et al., "An integrated QoS control architecture for IEEE 802.16 broadband wireless access systems", in Global Telecommunications Conference, GLOBECOM '05. IEEE, 2005, pp. 6 pp.-3335, 2005.

[17] IEEE Std 802.16e-2005. Part 16: "Air Interface for Fixed and Mobile Broadband Wireless Access Systems. Amendment 2: Physical and Medium Access Control Layers for Combined Fixed and Mobile Operation in Licensed Bands and Corrigendum 1", IEEE Computer Society, Feb. 2006.

[18] J. Y. Song, et al., "Hybrid Coupling Schemes for UMTS and Wireless LAN Interworking", AEU International Journal on Electronics and Communications, vol. 61, pp. 329-336, 2007.

[19] S. L. Tsao and C. C. Lin, "VGSN: A Gateway Approach to Interconnect UMTS/WLAN Networks", in Proc. of 13th IEEE International Symposium on Personal, Indoor, Mobile Radio Communications, 15-18 Sept. 2002, pp. 275-279.

[20] N. Vulic, I. Niemegeers, and S. H. de Groot, "Architectural Options for the WLAN Integration at the UMTS Radio Access Level”, in Proc. of IEEE 59th Vehicular Technology Conference, 17-19 May 2004, Milan, Italy, vol. 5, pp. 3009-3013.

[21] ITU-T Recommendation E.800. SERIES E: Overall network operation, telephone service, service operation and human factors, "Quality of services; concepts, models, objectives, dependability planning - Terms and definitions related to the quality of telecommunication services", 11-1988. 
[22] ITU-T Recommendation G.1000. SERIES G: Transmission systems and media, digital systems and networks, Quality of service and performance, "Communications quality of service: A framework and definitions". 11-2001.

[23] ITU-T Recommendation G.1010, SERIES G. Transmission systems and media, digital systems and networks, Quality of service and performance: "End-user multimedia QoS categories". 112001.

[24]3GPP TS 23.107 v6.1.0. "Technical Specification Group Services and systems Aspects, QoS Concept and architecture", 3GPP, 03-2004.

[25] 3GPP TS 23.207 v6.4.0. "Technical Specification Group Services and systems Aspects, End-toEnd QoS Concept and architecture", 3GPP, 09-2004. 